\title{
INTERFEROMETRY OF SOLAR FLARES
}

\section{AT 3-mm WAVELENGTH}

\author{
M. R. KUNDU, S. M. WHITE, N. GOPALSWAMY and J. LIM \\ Department of Astronomy, University of Maryland, College Park, MD 20742, U.S.A.
}

\begin{abstract}
We describe a set of millimeter interferometric observations of solar flares carried out in conjunction with GRO experiments during the 1991 June Campaign of the Max'91 Program. We show evidence that millimeter emission probes the most energetic $(\mathrm{MeV})$ electrons in solar flares; we also find that in the same flare there can be both impulsive nonthermal and gradual thermal millimeter emission. Millimeter emission usually occurs at the steep rise phase of the hard X-ray emitting electrons (25-100 KeV). There appears to exist some delay between BIMA mm-emission onset and GRO-BATSE $25-100 \mathrm{KeV} \mathrm{X}$-ray emission. Both results have implications for the particle acceleration process.
\end{abstract}

Key words: Sun: flares - Sun: particle emission - Sun: radio radiation

\section{Introduction}

Most millimeter-wave observations of solar flares have been hampered both by poor spatial resolution (because of the lack of synthesis interferometers) and by poor sensitivity (since the flux from the Sun's thermal emission is so high at millimeter wavelengths). The number of reported observations of bursts at millimeter wavelengths is relatively small, and there are none for which true high-spatial-resolution imaging data have been reported (see, e.g., White and Kundu 1992).

The usefulness of millimeter-wave observations lies in the fact that they are sensitive to both the highest energy electrons in flares as well as to cool material in the chromosphere. The emission of solar flares at millimeter wavelengths is of great interest both in its own right and because it is generated by the very energetic electrons which also emit gamma rays. Since high-resolution imaging at gamma-ray energies is not presently possible, millimeter observations can act as a substitute. In addition, the millimetric emission is undoubtedly optically thin (except possibly for a small class of very large flares which have spectra rising beyond $100 \mathrm{GHz}$; Kaufmann et al. 1985), and thus is not subject to the same ambiguities of interpretation which are prevalent in the study of solar microwave bursts. It can be used as a powerful diagnostic of the energy distribution of electrons in solar flares and its evolution, and of the magnetic field.

We have carried out high-spatial-resolution millimeter observations of solar flares using the Berkeley-Illinois-Maryland Millimeter Array (BIMA). At the present time BIMA consists of only three elements, which is not adequate for mapping highly variable solar phenomena, but is excellent for studies of the temporal structure of flares at millimeter wavelengths at several different spatial scales. The first dedicated observations of solar flares with a high-spatial-resolution millimeterwavelength interferometer were made by us in March 1989 (Kundu et al. 1990) when a solar active region with the greatest $\mathrm{X}$-ray flare production rate yet recorded appeared on the disk of the Sun. Our observations represented an improvement of an order of magnitude in both sensitivity and spatial resolution compared with previous solar observations at these wavelengths. We found that most of the flares occur- 
ring within the field of view during the March 1989 observations were detected at millimeter wavelengths, including both very impulsive and longer-duration events.

Based on these early observations it appeared that millimeter burst sources were not much smaller than microwave sources; generally source sizes were in excess of $2^{\prime \prime}$, but in some events sources may be $\sim 1^{\prime \prime}$. Thermal gyrosynchrotron models for the radio emission in the impulsive phase could be ruled out because the flux at millimeter wavelengths was too high.

\section{Observations During the June 1991 Campaign}

During the period of June 8-13, 1991, BIMA was in its most compact configuration. As mentioned earlier, the array presently has three antennas, providing three baselines. In effect each baseline provides a one dimensional spatial Fourier transform of the brightness distribution on the sky. For a point source the phase gives us positional information, while the amplitude is the total flux of the source. For an extended source the response of the interferometer depends on the relationship of the source dimension to the fringe spacing of the Fourier transform: only if the source dimension is smaller than about one-third of the fringe spacing do we expect the amplitude variability to correspond to the total flux in the source. For the 1991 June observations the fringe spacing ranged from $\sim 30^{\prime \prime} \mathrm{EW}$ to $\sim 45^{\prime \prime} \mathrm{NS}$; such large fringe spacings should guarantee that the correlated amplitude corresponds to the total flux of the source, at the expense of information about small spatial scales. These observations were made as part of the Max '91 Campaign involving the Compton Gamma Ray Observatory (GRO) along with other groundbased and space borne facilities.

\section{Results}

Figure 1 presents the GRO-BATSE (Burst and Transient Spectrometer Experiment) data along with $86 \mathrm{GHz}$ data for a burst observed on June 13, 1991, 17:5718:06 UT. The flare evolution is shown for the lowest BATSE energy channel, 25-50 $\mathrm{keV}$. In Figure 2 we have plotted the time profile of the BATSE 0.1-0.3 MeV channel and the BIMA $86 \mathrm{GHz}$ profile to the same scale. There were no significant counts above the background in the $0.3-1 \mathrm{MeV}$ channel. The remarkable feature of this flare is that while the overall profile in the $25-50 \mathrm{keV}$ range is relatively simple, with a sharp rise to a plateau level containing a number of sub-peaks followed by a rapid decay, in the higher-energy channels we see a sharp spike right at the beginning of the flare. We and others (Ramaty 1969; Ramaty and Petrosian 1972) have argued that millimeter emission in the impulsive phase should be due to gyrosynchrotron emission from the most energetic electrons in a flare, and on this basis one would predict that the millimeter emission would also show a sharp spike in the onset phase. This is exactly what is seen. The close similarity of the 0.1-0.3 MeV and $86 \mathrm{GHz}$ profiles provides convincing evidence that the millimeter emission is indeed due to the most energetic electrons in a solar flare: the same energetic electrons that produce gamma ray continuum. The importance of this result is that we can obtain high-spatial-resolution information on source structures 


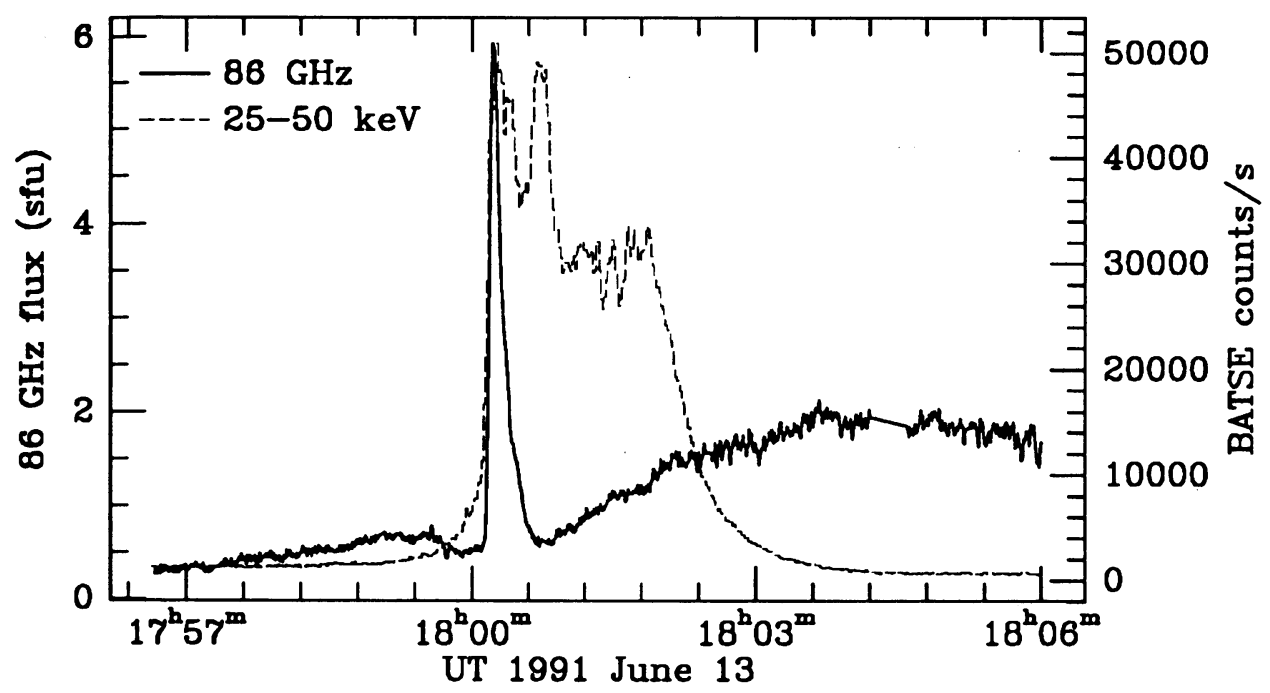

Fig. 1. Comparison of the time profiles of the $1991 \mathrm{June} 1318$ UT flare emission in the 25-50 keV hard X-rays seen by BATSE (broken line) and the $86 \mathrm{GHz}$ emission recorded by BIMA (solid line).

with millimeter-interferometer observations, which is not presently possible directly with gamma-ray observations.

Figure 1 shows further that the sharp rise in the $25-50 \mathrm{keV}$ range coincides exactly with the $86 \mathrm{GHz}$ rise, although there is some emission in the $25-50 \mathrm{keV}$ range just prior to the impulsive rise which has no signature at $86 \mathrm{GHz}$. However, unlike the $25-50 \mathrm{keV}$ burst which has a plateau lasting $\sim 3$ min and containing another major peak, the mm emission drops abruptly to almost its pre-burst level. This figure also emphasizes that, in addition to the impulsive phase coinciding with the gamma ray emission, the millimeter emission shows an extended component which rises slowly and continues beyond 1806 UT. Thus in this event we clearly see two easily-distinguishable components in the millimeter emission: an impulsive component coinciding in time with the impulsive gamma ray emission, and a gradual extended component with no gamma ray counterpart. We also note the small dip in the $86 \mathrm{GHz}$ emission immediately prior to the impulsive rise, which seems to be a common feature of pre-flare activity in millimeter-wave emission.

Figure 3 shows an event in which impulsive $25-50 \mathrm{keV}$ emission was seen, but there was very weak impulsive-phase emission at $86 \mathrm{GHz}$, even though the count rates at 25-50 keV were well above the levels of some small events which produced both $25-50 \mathrm{keV}$ and $86 \mathrm{GHz}$ impulsive-phase emission. The $86 \mathrm{GHz}$ profile in this case shows gradual mm burst emission corresponding roughly with the GOES soft $\mathrm{X}$-ray emission, although the BATSE data show several sharp peaks. In this case the hard X-ray rise corresponds to the rise phase of both $\mathrm{mm}$ and soft $\mathrm{X}$-ray emission. A number of 1991 June flares, both with and without $86 \mathrm{GHz}$ impulsive phases, 


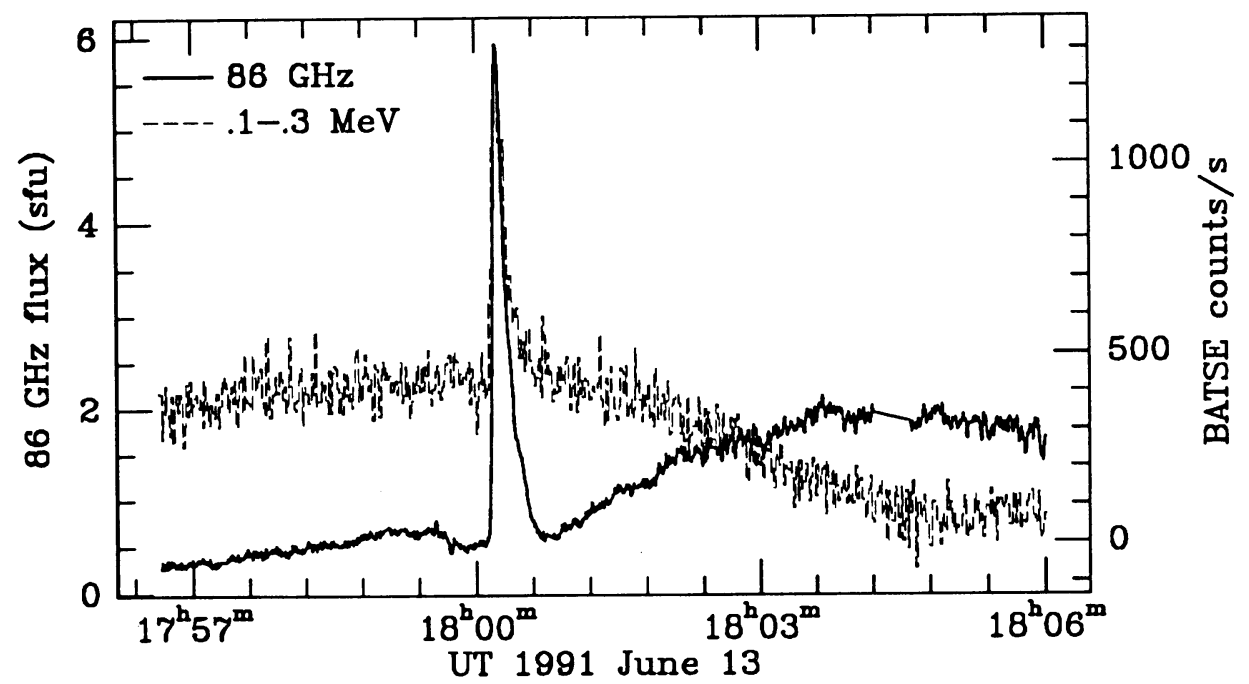

Fig. 2. Comparison of the time profiles of the 1991 June 1318 UT flare emission in 0.1-0.3 $\mathrm{MeV}$ hard $\mathrm{X}$-rays (broken line) and the $86 \mathrm{GHz}$ emission (solid line).

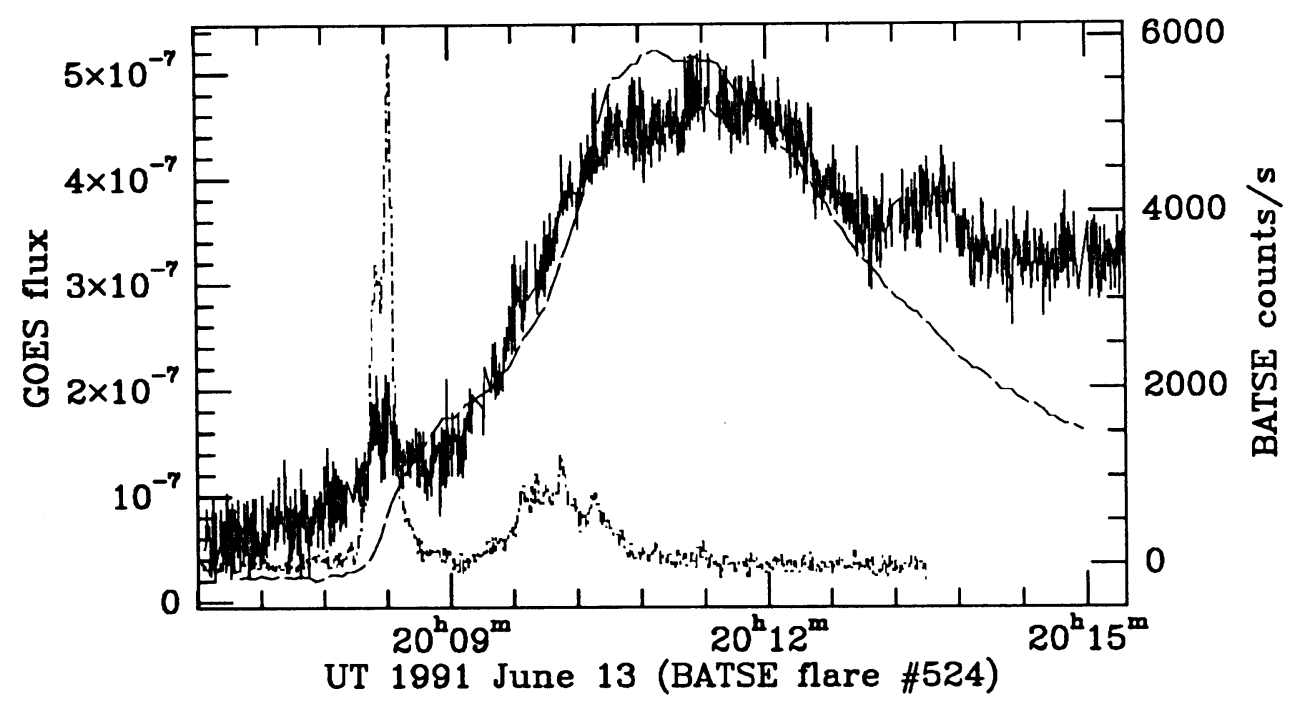

Fig. 3. The evolution of emission for a flare at around 20 UT on 1991 June 13 seen in soft $\mathrm{X}$-rays recorded by the GOES satellite (8-25 keV; long-dashed line), in 25-50 keV hard $\mathrm{X}$-rays (BATSE data, short-dot-dashed line) and at $86 \mathrm{GHz}$ (BIMA data, solid line). 
showed a thermal phase at $86 \mathrm{GHz}$ which was similar in profile to the GOES data, including the June 1318 UT event. We also found evidence that for some simple impulsive events with a very sharp rise there is a clear delay of several seconds between the 25-50 keV impulsive rise and the $86 \mathrm{GHz}$ rise. Since we can associate the $86 \mathrm{GHz}$ emission with high-energy electrons (>300 keV), the implication is that there is a delay between the production of the $25-50 \mathrm{keV}$ electrons and the $>300$ $\mathrm{keV}$ electrons. Such delays can be attributed to the need to accelerate electrons from low to high energies, and can be used to constrain candidate emission mechanisms.

\section{Conclusions}

Our 1991 June observations permit us to make the following general conclusions.

1) There are two phases in millimeter burst emission: a nonthermal impulsive phase and a thermal gradual phase. Both phases are often observed in the same flare.

2) Impulsive-phase millimeter burst emission probes $\mathrm{MeV}$ electrons produced during flares. Some flares show no nonthermal impulsive phase at millimeter wavelengths, although they seem to show it at $25-50 \mathrm{keV}$. These gradual phase flares correspond well to the GOES soft X-ray emission.

3) Millimeter emission usually occurs at the steep rise phase of the hard Xray emitting electrons (25-100 keV). There appears to exist some delay between BIMA mm-emission onset and BATSE 25-100 keV X-ray emission. Both results have implications in the particle acceleration process.

\section{Acknowledgements}

This work was made possible by GRO Phase I Guest Investigator grant NASANAG-W-1541. Solar research at Maryland is also supported by NSF grant ATM 90-19893 and NASA grants NAG-5-1540, and NAG-W-2172. Scientific research at U. Md. with BIMA is supported by NSF grant AST 91-00306.

\section{References}

Kaufmann, P., Correia, E., Costa, J.E.R., Vaz, A.M.Z., Dennis, B.R.: 1985, Nature, 313, 380

Kundu, M.R., White, S.M., Gopalswamy, N., Bieging, J.H., Hurford, G.J.: 1990, Astrophys. J. (Letters), 358, L69.

Ramaty, R.: 1969, Astrophys. J., 158, 753.

Ramaty, R., Petrosian, V.: 1972, Astrophys. J., 178, 241.

White, S.M., Kundu, M.R.: 1992, Solar Phys., in press. 